DOCUMENTS

pour l'histoire

des techniques
Documents pour l'histoire des techniques

Nouvelle série

$17 \mid 1^{\text {er }}$ semestre 2009

L'invention technique et les figures de l'inventeur (XVIII $-X^{\mathrm{e}}$ siècles)

\title{
La mesure de l'homme. Instruments et tests du musée d'histoire naturelle de Lille
}

Paris, Somogy éditions d'art/Lille, Musée d'histoire naturelle, ville de Lille, 2002, 262 pages.

Pierre Savaton

\section{(2) OpenEdition}

\section{Journals}

Édition électronique

URL : http://journals.openedition.org/dht/614

DOI : $10.4000 /$ dht.614

ISSN : 1775-4194

Éditeur :

Centre d'histoire des techniques et de l'environnement du Cnam (CDHTE-Cnam), Société des élèves du CDHTE-Cnam

Édition imprimée

Date de publication : 31 mars 2009

Pagination : 219-220

ISBN : 978-2-95-30779-3-3

ISSN : 0417-8726

Référence électronique

Pierre Savaton, «La mesure de l'homme. Instruments et tests du musée d'histoire naturelle de Lille », Documents pour I'histoire des techniques [En ligne], 17 | $7^{\text {er }}$ semestre 2009, mis en ligne le 27 septembre 2010, consulté le 21 septembre 2020. URL : http://journals.openedition.org/dht/614 ; DOI : https:// doi.org/10.4000/dht.614

Ce document a été généré automatiquement le 21 septembre 2020.

(C) Tous droits réservés 


\section{La mesure de l'homme. Instruments et tests du musée d'histoire naturelle de Lille}

Paris, Somogy éditions d'art/Lille, Musée d'histoire naturelle, ville de Lille, 2002, 262 pages.

Pierre Savaton

\section{RÉFÉRENCE}

La mesure de l'homme. Instruments et tests du musée d'histoire naturelle de Lille, Paris, Somogy éditions d'art/Lille, Musée d'histoire naturelle, ville de Lille, 2002, 262 pages. 
1 Ce catalogue prolonge l'exposition de 2002

$\mathrm{du}$ Musée d'Histoire naturelle et d'ethnographie de Lille sur La mesure de l'homme à travers plus de 150 instruments et tests. Lille possède probablement la plus grande collection d'instruments et de tests utilisés du XIX ${ }^{\mathrm{e}}$ au $\mathrm{XX}^{\mathrm{e}}$ siècles pour mesurer l'homme et plus précisément, audelà de sa force et de son acuité visuelle ou auditive, mesurer sa mémoire, ses temps de réaction, sa psychomotricité, ses aptitudes et raisonnements, son intelligence. Ces 200 pièces collectées par

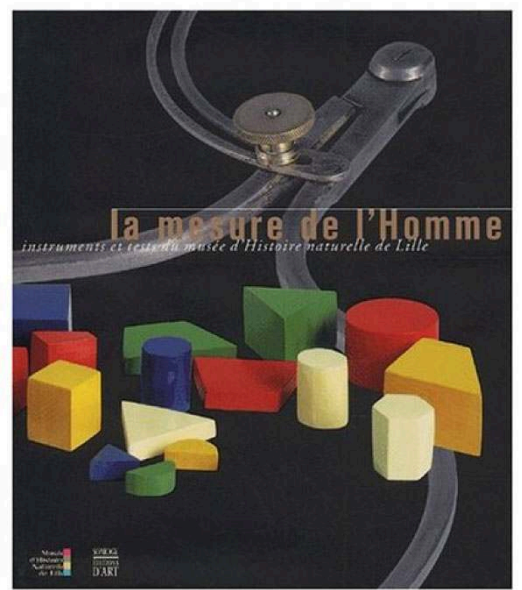
Jean Cnudde, ancien directeur du CIO de Lille, construites pour la plupart entre 1900 et 1950, mais remontant parfois au début $\mathrm{du} \mathrm{XIX}^{\mathrm{e}}$ siècle, illustrent les débuts de la psychologie expérimentale et de ses applications en orientation et sélection professionnelle.

2 La jaquette noire de ce très bel ouvrage nous accueille avec un compas (thoracique) de la fin du XIX ${ }^{\mathrm{e}}$ et un test clinique de Vygostki, Hanfmann et Kasanin de 1921. Un compas de métal froid, dont la photographie révèle l'aspect encore brut et rudimentaire, aux pointes masquées et laissées ainsi à notre imaginaire, enserre un jeu de formes en bois, colorées de teintes vives et rassurantes comme un jeu d'enfant. D'emblée les auteurs nous replacent par l'esthétique dans l'histoire ambiguë de cette mesure de l'homme où idéologie de la norme physique et sociale et tentation de l'eugénisme, côtoient quête de progrès social et recherche d'un développement harmonieux de l'enfant. Ces instruments et machines, bien souvent énigmatiques, ne pouvaient être placés sous nos yeux sans un minimum d'explications et de mise en contexte. C'est le choix des auteurs traduit dans cet ouvrage par une découpe en deux parties : un ensemble d'articles pour brosser une histoire de ces instruments et des idées qui les ont générés et portés, puis un catalogue de 166 objets de l'exposition.

3 Jean Cnudde, à l'origine de cette collection, fixe quelques repères historiques et épistémologiques. Il évoque ainsi successivement la naissance précoce de la sensorimétrie avec la détermination des seuils de sensation de Weber et Fechner en 1830, l'émergence de la psychologie expérimentale avec l'équation personnelle (temps de réaction) d'Exner en 1868, le Tapping-test (premier test psychomoteur) de Kraepelin en 1896 et l'essor de la psychologie de l'enfant à partir de 1905, sous l'impulsion de Binet et Simon. À chaque fois, l'accent est mis sur le matériel et un renvoi est fait au catalogue. C'est directement à travers les objets des laboratoires que Françoise Parrot nous présente la différence d'approche expérimentale entre la psychologie française, russe et américaine. Les premiers psychologues français, tous médecins et positivistes, ont cherché à contourner la tradition spiritualiste du XIX ${ }^{\mathrm{e}}$ par le matérialisme de la physiologie en s'appuyant sur les méthodes de la médecine expérimentale. C'est une psycho-physiologie qui s'est développée et cet ancrage dans la médecine a conduit à aborder le normal par le pathologique. La psychologie anglo-saxonne, darwinienne, s'est appuyée en revanche fondamentalement sur l'animal et l'expérimentation animale. Entre l'intelligence de l'homme et celle de l'animal, il n'y a alors qu'une différence de degré et pas de nature. Les laboratoires américains sont pour cela d'abord 
des animaleries. La psychologie française, lamarckienne, fit de l'hérédité des caractères acquis un argument républicain.

4 Francis Danvers replace cette mesure de l'homme dans le cadre de l'orientation professionnelle des années 1920-1930 en France, portée par la loi Astier de 1919 relative à l'organisation de l'enseignement technique, industriel et commercial. Marcel Turbiaux prolonge cet éclairage en s'arrêtant sur le parcours de J.-M. Lahy (1872-1943), fondateur de la psychotechnique en France. Son texte est un véritable guide qui nous dévoile comment la physiologie et la psychologie se sont attachées aux questions de l'organisation du travail et pour cela à la description psycho-physiologique des tâches, à la sélection et à la formation en conséquence des travailleurs. Les idées politiques et économiques ne sont pas étrangères au développement de la psychologie et l'on voit pourquoi Lahy condamna le taylorisme, qu'il accusa de méthode de surmenage, pour sa détermination uniquement physiologique et son ignorance de la dimension psychologique du travail. Discours de progrès social et discours sur l'exploitation sociale renvoient aux apports de la psychotechnique. Charles Thomas, après avoir passé en revue les instruments et tests de l'examen psychotechnique, attire notre attention sur leur remplacement à la fin du $\mathrm{XX}^{\mathrm{e}}$ siècle par les tests papier-crayon, puis par les interviews.

5 Michel Hutteau aborde la question tant attendue de la mesure de l'intelligence et montre comment les tests $\mathrm{du}$ début $\mathrm{du} \mathrm{XX}^{\mathrm{e}}$ siècle marquèrent un renouveau paradigmatique par rapport aux tests associationnistes de la fin du XIX ${ }^{e}$ siècle issus d'une psychologie différentielle imprégnée dans ses visées d'un darwinisme social. De l' échelle métrique de l'intelligence de Binet-Simon, destinée à identifier des déficiences mentales, au calcul d'un âge mental, puis d'un quotient intellectuel, on suit l'évolution de ces tests, de l'école à la sélection des G.I. en 1917. Ces tests dont plusieurs sont figurés dans le catalogue, sont restés secondaires dans le système éducatif français à la différence des États-Unis et de la Grande-Bretagne où ils jouent un rôle important dans la sélection et l'orientation scolaire.

6 Stéphane Callens clôt cette première partie de l'ouvrage par une réflexion sur les ambiguïtés des visées et applications de ces mesures de l'homme. Si 1865 marque avec Claude Bernard la revendication d'une médecine expérimentale, dont la psychologie naissante tira partie, cette date représente aussi pour l'auteur une bascule dans les arguments des avocats de l'esclavage, dont Paul Broca et sa craniométrie. La défaite de la sécession des états esclavagistes du sud des États-Unis en 1865 fit passer le débat sur l'égalité ou l'inégalité des hommes du champ de la religion, de l'économie et du droit à celui de biologie et de l'anthropologie. À la psychologie naissante et ses premiers pas dans la mesure de l'homme s'est ajoutée la perversion idéologique d'une craniométrie destinée à justifier par des différences de mesures une hiérarchie entre humains et l'élimination physique de certaines ethnies. Callens nous rappelle également comment Alexis Carrel proposa au gouvernement de Vichy de remplacer le certificat d'études par un inventaire physiologique, intellectuel et moral destiné à classer la jeunesse en catégories déterminant leur position sociale. Avec Broca et Carrel, la mesure de l'homme devenait un instrument au service de l'élimination des plus faibles. Le régime nazi conduisit cette malmesure jusqu'à l'horreur et Nuremberg en tira deux conditions incontournables à l'expérimentation sur l'homme. Le propos s'arrête avec 1950 mais on ne peut s'empêcher de penser à l'exploitation criminelle que d'autres régimes 
tortionnaires ont $\mathrm{pu}$ faire après cette date des apports des mesures sur les comportements humains individuels par exemple.

7 Mais, si ambiguïté il y a, c'est justement parce que les visées normales de cette mesure de l'homme ne nourrissaient pas de telles intentions, bien au contraire, comme nous l'ont montré les articles précédents.

8 Notons enfin la très belle illustration de toute cette première partie, faite d'images pleine page d'instruments et de tests, mais aussi de témoignages photographiques de la mise en œuvre de ces matériels.

9 La partie catalogue de cet ouvrage expose enfin tous ces instruments et tests, servis par de superbes photographies en plan rapproché sur fond blanc. Ils y sont classés par discipline de mesure : anthropométrie, dynamométrie, mesures respiratoires, mesures cardio-vasculaires, sensorimétrie... mesure des aptitudes, de l'apprentissage, du caractère et de la personnalité. Ils sont accompagnés, sauf exceptions, d'une notice explicative sur leur fonctionnement ou leur principe. Mais, celle-ci ne peut répondre aux interrogations éventuelles du lecteur-visiteur sur les conclusions que les expérimentateurs tiraient des mesures et résultats ainsi obtenus et sur les conséquences qui en découlaient pour le sujet qui s'était prêté à ces mesures. La question de l'usage conserve à ces objets un aspect énigmatique, voire parfois inquiétant.

10 À feuilleter les pages consacrées aux facultés motrices et aux tests de mesure de l'intelligence ou des apprentissages, on croise des instruments et tests (dextérimétrie, batterie motrice et d'habileté manuelle, planches à encastrement, compléments d'images, jeux de Kim), qui ne sont pas sans rappeler d'actuels jeux pour enfants destinés à développer telle ou telle aptitude (mémoire, agilité, rapidité, etc.).

11 Un livre à lire et à regarder pour un voyage historique et pour une réflexion sur les liens entre instruments et sciences, sciences et société.

\section{AUTEUR}

PIERRE SAVATON

IUFM, Université de Caen Basse-Normandie 